\title{
Effects of nucleus posterior thalami lesions on the performance of visual discriminations'
}

MICHAEL PETERS ${ }^{2}$ and R. M. COOPER, The University of Calgary, Calgary, Alberta, Canada

Lesions in the nucleus posterior thalami were found to differentially affect performance of a black vs white and $a$ stripes discrimination. Performance of a difficult brightness discrimination was more adversely affected by nucleus posterior thalami lesions than performance of an easy brightness discrimination. The findings are interpreted in terms of sensory and motivational disturbances.

Thompson (1965) suggested that the nucleus posterior thalami (npt) is part of a system concerned with the elaboration of memory traces for simple visual habits. The principal evidence for this was the Thompson \& Rich (1962) finding that npt lesions appear to affect the retention of a black vs dark-grey discrimination more severely than the retention of a horizontal vs vertical stripes (pattern) discrimination.

Unfortunately, Thompson \& Rich (1962) used percentage savings scores to evaluate pre- and postoperative performances of npt-lesioned animals. Use of this measure can lead to certain difficulties. For instance, npt lesions might produce a general postoperative disturbance which equally affects all animals and which causes a certain number of postoperative errors to be made by all animals. One would expect, in such a case, that the percentage savings scores of those animals which made many preoperative errors will appear superior to those of animals which made few preoperative errors. As Thompson and Rich's black vs dark-grey group made fewer preoperative errors than their pattern group, even though the postoperative error scores were similar for both groups, the reported differences in retention after npt lesions may be a measurement artifact. Part of this study was designed to test such a possibility. In addition, effects of npt lesions on a variety of visual discriminations were studied.

\section{METHOD}

The Ss were 40 naive male Long Evans black-hooded rats averaging $250 \mathrm{~g}$. A modified Yerkes box, similar to that described by Thompson \& Bryant (1955), was used. Ss were run under shock motivation; approaches to the incorrect stimulus card were punished by a $.8 \mathrm{~mA}$ shock. After pretraining Ss were given 10 trials daily, on the discriminations described below, until a criterion of three or less errors over 30 consecutive trials was reached.

Discrimination A: Light card positive vs black card negative. The light card was a translucent Plexiglas sheet covered with transparent white paper. A 75-W light attached behind the goal box was the only light source; the black card did not transmit any light. $N=6$. $B$ : As in $A$ but black card positive vs light card negative. $N=6$. $C$ : White card positive vs black card negative. The brightness of the background against which the stimulus cards were displayed was manipulated in pilot studies until Ss made approximately as many errors in learning Task $\mathrm{C}$ as were made by Ss learning Task $F$. $N=7$. D: White card positive vs medium grey card negative. $\mathrm{N}=8$. $\mathrm{E}$ : Grey stimulus card with horizontal $19-\mathrm{mm}$ white stripe positive vs grey stimulus card with horizontal 19-mm black stripe negative. $\mathrm{N}=3$. F: White card with $12-\mathrm{mm}$ black horizontal stripes positive vs white card with $12-\mathrm{mm}$ vertical stripes negative. The stripes were $12 \mathrm{~mm}$ apart. $\mathrm{N}=10$.

The stimulus cards in Tasks C, D, E, and F were illuminated by a $100-W$ overhead light situated $50 \mathrm{~cm}$ from the stimulus cards. Within three days after reaching criterion the animals received bilateral lesions of the npt. Electrode placements, using de Groot's (1959) atlas, were $1.5 \mathrm{~mm}$ laterally from the midline, $3 \mathrm{~mm}$ anterior to frontal zero, and $4.8 \mathrm{~mm}$ beneath the dura. Lesions were produced at $2 \mathrm{~mA}$ for $20 \mathrm{sec}$. Postoperative training, to the preoperative criterion, commenced 10 days after surgery. As control Ss exhibited uniform perfect retention after 10 days only the scores of experimental Ss will be given.

\section{RESULTS}

Histology revealed that the lesions had been well placed within the npt region. Most of the Ss displayed pupillary dilation which appeared to be permanent. No obvious correlation between degree of pupillary dilation and error scores was found. Some Ss, with lesions in the more posterior portion of the npt region, showed an upward flexion of the head which disappeared a few days after surgery. All Ss began to show a strong reaction to shock after they had made a few postoperative errors. This manifested itself in a reluctance to leave the startbox, freezing and lack of spontaneous behavior in the testing apparatus. Postoperatively, Ss seemed often unable to avoid the incorrect stimulus card and persisted in approaching it despite shock.

Pre- and postoperative performances are presented in Fig. 1. Comparison of Groups $\mathrm{C}$ and $\mathrm{F}$ indicates that their preoperative learning scores were successfully equated. Their postoperative scores, however, differed significantly (MannWhitney $U$ test, $U=10$ ). Postoperative performance deteriorated through Tasks A to E. None of the animals in Group E was able to perform the discrimination postoperatively. In contrast, Ss in Groups A and B made significantly fewer (combined scores for $\mathrm{A}$ and $\mathrm{B}, \mathrm{t}=6.7, \mathrm{~N}=12$ ) errors on the first 10 postoperative trials than on the first 10 preoperative trials. This suggests that the easier discriminations $A$ and $B$ are less affected by $n p t$ lesions than the difficult discrimination $E$; this stands in contradiction to Thompson's (1965) model.

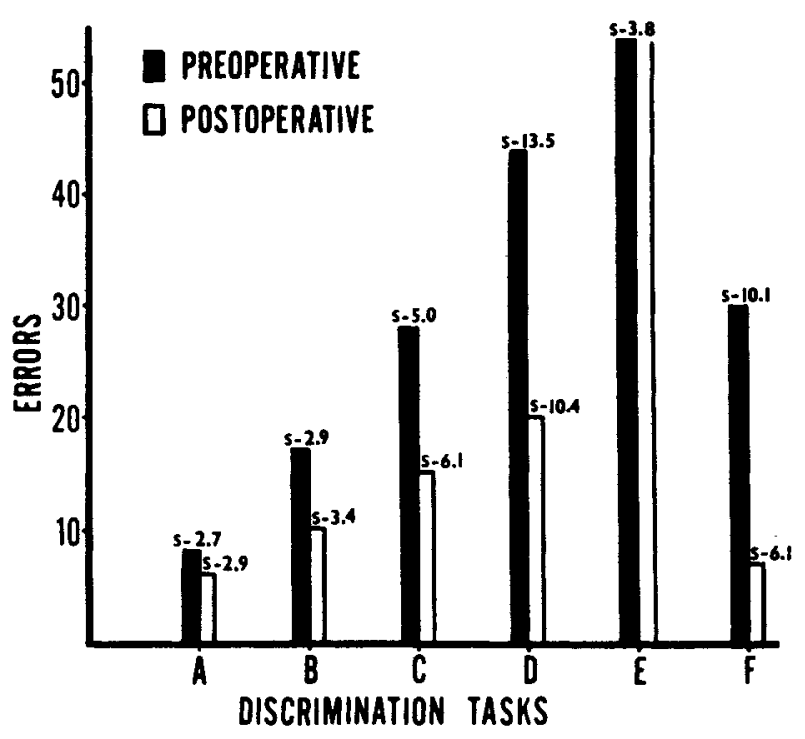

Fig. 1. Pre- and postoperative performances of npt-lesioned animals. None of the Ss in Group E was able to perform the task postoperatively ( $s=$ standard deviation $)$. 
information on optimal test features. Some improvement in test performance may occur as these features are uncovered and implemented in future research.

One feature which may be identified now, however, concerns the distance between the relevant stimulus cue, i.e., the borders of the stimulus figures, and the locus at which the dolphin made its pressing response. Wela tended to press the contour-stimuli at or near their centers, which were uniform in appearance across stimuli, and offered no relevant cue. Thus, there was spatial discontiguity between locus of the relevant cue at the borders and locus of the pressing response. Meyer et al (1965) report that displacement of the relevant cue by as little as .5 in. from the locus of response results in considerable drop in discrimination-learning set performance in monkeys. Stollnitz (1965) has provided a theoretical basis for this decrement in terms of a failure to reinforce the observing response, i.e., the response of attending to the relevant cue, in problems characterized by spatial discontiguity and by relatively few trials. He notes that monkeys tend to look where they put their fingers, and animals lacking fingers look at what their noses touch. If the observing response is not strengthened, then within-problem learning is retarded and transfer of learning across problems is precluded. In this case, some irrelevant stimulus dimension, such as spatial position, may come to control behavior.

Examination of the degree of spatial discontiguity for the contour pairs used in the first 40 12-trial problems of the present study provided a partial check on the assumption that this variable was an important determinant of the dolphin's performance level. In 14 of the 40 problems, for Trials 2 to 12 , discrimination performance-though not necessarily learning-could be classified as "good." These were problems in which either the $\mathbf{S}+$ stimulus was chosen in at least 9 of the 11 trials $(p<.06)$, or the $S$ - stimulus was so chosen. The remaining 26 problems were classified as "poorer" discrimination performance. Measurements taken of the minimum distance from the center of each contour stimulus to its border revealed that the average minimum distance was smaller by .6 in. for the "good" problems. As has been noted, this is a sufficient spatial discontiguity to have detrimental effects on primate performance.

It appears, therefore, that there are both empirical and theoretical grounds for concluding that spatial discontiguity between stimulus-cue locus and response locus could have been of major importance in restricting the dolphin's performance level in this study. Research is currently in progress to investigate this variable and to assess further the dolphin's ability to form learning sets.

\section{REFERENCES}

KELLOGG, W., \& RICE, C. Visual problem-solving in a Bottlenose Dolphin. Science, 1964, 143, 1053-1055.

MEYER, D. R., TREICHLER, F. R., \& MEYER, P. M. Discrete-trial training and stimulus variables. In A. M. Schrier, H. F. Harlow, and F. Stollnitz (Eds.), Behavior of nonhuman primates. Vol 1. New York: Academic Press, 1965. Chap. 1.

MILES, R. C., \& MEYER, D. R. Learning sets in marmosets. Journal of Comparative \& Physiological Psychology, 1956, 49, $219-222$.

NORRIS, K. S. (Ed.) Whales, dolphins, and porpoises. Berkeley: University of California Press, 1966.

STOLLNITZ, F. Spatial variables, observing responses, and discrimination learning sets. Psychological Review, 1965, 72, 247-261. NOTE

1. This research was supported in part by a grant from Oceanic Institute, Oahu. Appreciation is extended to Sea Life Park, Oahu, for generous use of their facilities.

\section{(Continued from page 97)}

\section{DISCUSSION}

Thompson \& Rich's (1962) basic finding, that of differential performance on black vs dark-grey (brightness) and pattern discriminations after npt lesions, was confirmed. The differences do not originate through measurement artifact.

The postoperative reaction to shock, as observed in this study, suggests that at least some portion of the postoperative deficit may be attributed to the level of shock used. Further evidence for the importance of motivational factors was provided by Breen \& Thompson (1966), who have shown that postoperative deficits of npt-lesioned animals are minimal if they are tested under thirst motivation and Thompson et al (1967) who, using mild shock, reported results contradicting both this and Thompson \& Rich's (1962) study. Also of relevance might be Myers' (1964) report that lesions in the posterior thalamic area in the cat produce marked visual unresponsiveness.

In addition to motivational factors, sensory factors, possibly interacting with the former to produce postoperative deficits, must also be involved in npt-lesion effects. This is indicated by the increasing difficulties Ss had in solving brightness discriminations of increasing difficulty (Tasks $A$ to $E$ ) postoperatively. It might be argued that the postoperative errors simply reflect the difficulty Ss had in learning the discrimination originally; this is negated by the performance of Groups $\mathrm{C}$ and $\mathrm{F}$ and by the fact that all control Ss, regardless of the discrimination, showed perfect retention over the postoperative recovery interval. Thus, the differential performance on Tasks $\mathrm{C}$ and $\mathrm{F}$ might be interpreted as reflecting a sensory disturbance which somehow affects $\mathrm{C}$ more adversely than F. Support for such a possibility is provided by the finding that naive npt-lesioned Ss trained to learn either $\mathrm{C}$ or $\mathrm{F}$ also show the differences described previously for the training-operation-retraining situation (Peters, M., unpublished, $\mathrm{N}_{1}=5, \mathrm{~N}_{2}=6, \mathrm{p}=.015$, by Mann-Whitney $\mathrm{U}$ test; stripes performance superior).

The above interpretation of npt lesion deficits in terms of motivational and sensory factors stands in contrast to Thompson's (1965) stress on memory factors. It is felt, however, that before elusive factors such as memory are considered, the role of the more tangible motivational and sensory factors in $\mathrm{npt}$ lesion deficits should be thoroughly explored. Currently, attempts are made to experimentally define those stimulus dimensions which differentiate between discriminations $\mathrm{C}$ and $\mathrm{F}$. If such stimulus dimensions can be defined it might be possible to deduce the sensory impairments following npt lesions.

\section{REFERENCES}

BREEN, T., \& THOMPSON, R. Cortical and subcortical structures mediating a visual conditioned response motivated by thirst. Journal of Comparative \& Phy siological Psychology, 1966, 61, 146-150.

De GROOT, J. The rat forebrain in stereotax ic coordinates. Amsterdam: N.V. Nord-Hollandsche Uitgevers, Maatschappij, 1959.

MYERS, R. E. Visual deficits after lesions of the brain stem tegmentum in cats. Archives of Neurology, 1964, 11, 73-90.

THOMPSON, R. Centrencephalic theory and inter-hemispheric transfer of visual habits. Psychological Review, 1965, 72, 385-398.

THOMPSON, R., \& RICH, I. Differential effects of posterior thalamic lesions on retention of various visual habits. Journal of Comparative $\&$ Physiological Psychology, 1963, 56, 60-65.

THOMPSON, R., LUKASZEWSKA, I., SCHWEIGERDT, A., \& McNEW, $J$. Retention of visual and kinesthetic discriminations in rats following pretecto-diencephalic and ventral mesencephalic damage. Joumal of Comparative \& Physiological Psychology, 1967, 63, 458-468. NOTES

1. This study was supported by NRC Grant APA-135.

2. Now at University of Western Ontario, London, Ontario, Canada. 